\title{
Author Correction: A technology-forcing approach to reduce nitrogen pollution
}

David R. Kanter and Timothy D. Searchinger

Correction to: Nature Sustainability https://doi.org/10.1038/s41893-018-0143-8, published online 15 October 2018.

In the version of this Perspective originally published, in the last paragraph of the section 'The US corn sector', there was an incorrect mention of Fig. 3; it should have referred to Fig. 2. This has now been corrected. The Supplementary Information has also been updated to provide absolute numbers for avoided $\mathrm{N}$ pollution as calculated in the main text. 